\title{
SPORTS ANTHROPOLOGICAL AND SOMATOTYPICAL COMPARISON BETWEEN MALE WRESTLERS AND HAPKIDOIN OF DIFFERENT PERFORMANCE LEVELS
}

\author{
Christoph Raschka, Jasmin Müller \\ Institute of Sports Sciences, Julius Maximilians University Würzburg, Germany
}

\begin{abstract}
The present study compares the anthropometry and somatotyping of $\mathrm{n}=40$ healthy male wrestlers (age range 15-37 years) and $n=40$ Hapkidoin (age range 23-29 years) with respect to various performance categories.

On average, the mean age of wrestlers was lower, compared to hapkidoin. The wrestlers of both performance classes are considerably smaller (mean height $170 \mathrm{~cm}$ ) than the hapkidoin (high performance class $176.5 \mathrm{~cm}$, lower performance class $180 \mathrm{~cm}$ ). When comparing the weight classes of the wrestlers, the average body height increases continuously with increasing weight class in conjunction with most other anthropometric parameters.

In the chessboard pattern graphic to Conrad, the hapkidoin appear rather moderately pyknomorphic as well as slender, the wrestlers are moderately leptomorphic and metrosome as well as smaller.

In Parnell's somatochart, the wrestlers have an average somatotype of 5 $3-3$, the hapkidoin of $5-3-4$. In the somatochart of Heath \& Carter, an average somatotype of $6-3-2$ is shown for both combat sport collectives, whereby the wrestlers tend to the endomorphic axis.
\end{abstract}

Keywords: sports anthropology; comparison; Hapkido; wrestling; male athletes

\section{INTRODUCTION}

Wrestling represents one of the oldest forms of combat. Babylonian and Egypt reliefs show wrestlers using most of the holds known in the present-day sport. Hapkido (also Hap Ki Do) is a Korean martial art, which originated in Japanese Daitō-ryū Aiki-jūjutsu. Through the influence of other fighting styles 
to Hapkido it developed into an independent martial art that is coined by a comprehensive curriculum.

\section{PARTICIPANTS AND METHODS}

The present study compares the anthropometry and somatotyping of $\mathrm{n}=40$ healthy male wrestlers (age range 15-37 years) and $n=40$ Hapkidoin (age range 23-29 years) with respect to various performance categories.

Each proband participated voluntarily and the data were used anonymously. Anthropometric data and computed constitutional and somatotypical parameters in this work correspond to international standards (Conrad 1963, Heath \& Carter 1967+1990, Knußmann 1996, Martin \& Knußmann 1988, Raschka 2006, Tittel \& Wutscherk 1972). The analysis of differences was tested by ANOVA.

\section{RESULTS}

The distribution of constitutional types after Conrad (1963) and the somatotypes after Parnell (1954) and Heath \& Carter (1967) are summarized in Figures 1-3.

a

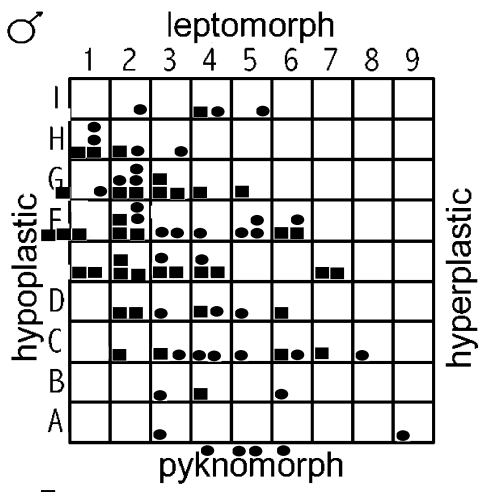

Wrestling b $ఠ$

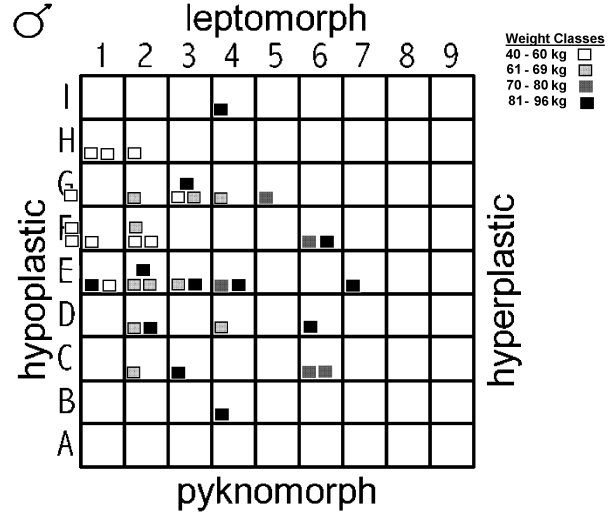

Figure $1 \mathrm{a}+\mathrm{b}$. Male constitutional types of wrestlers and Hapkido fighters in the chessboard pattern graphic after Conrad (1963): a) first chessboard graphic: wrestling vs. Hapkido; b) second chessboard graphic: wrestling differentiated according to weight classes. 
a
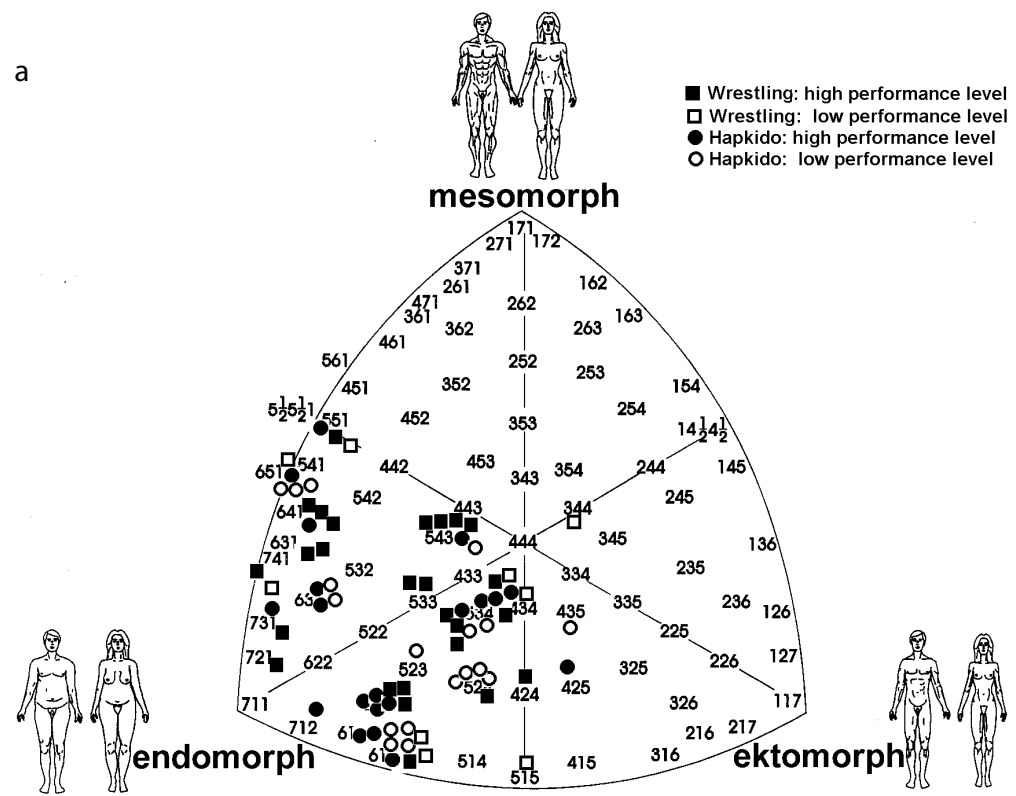

b
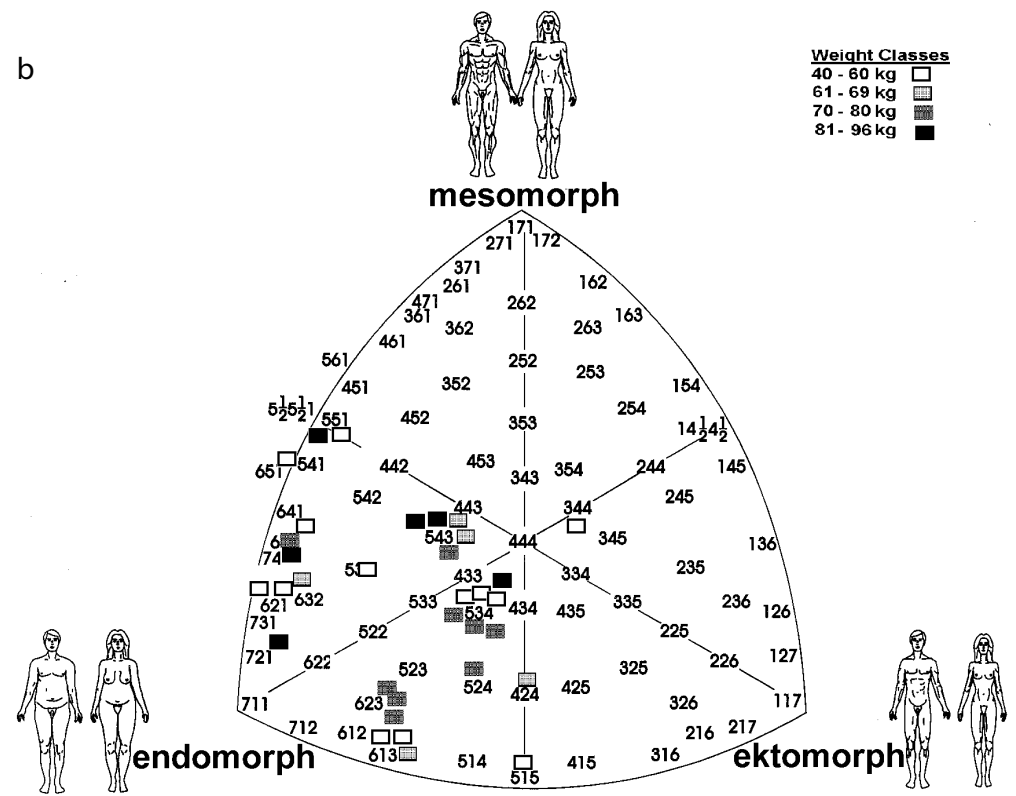

Figure $\mathbf{2 a}+\mathbf{b}$. Male constitutional types of wrestlers and Hapkido fighters in the somatochart after Parnell (1954): a) first somatochart: wrestling vs. Hapkido; b) second somatochart: wrestling differentiated according to weight classes. 

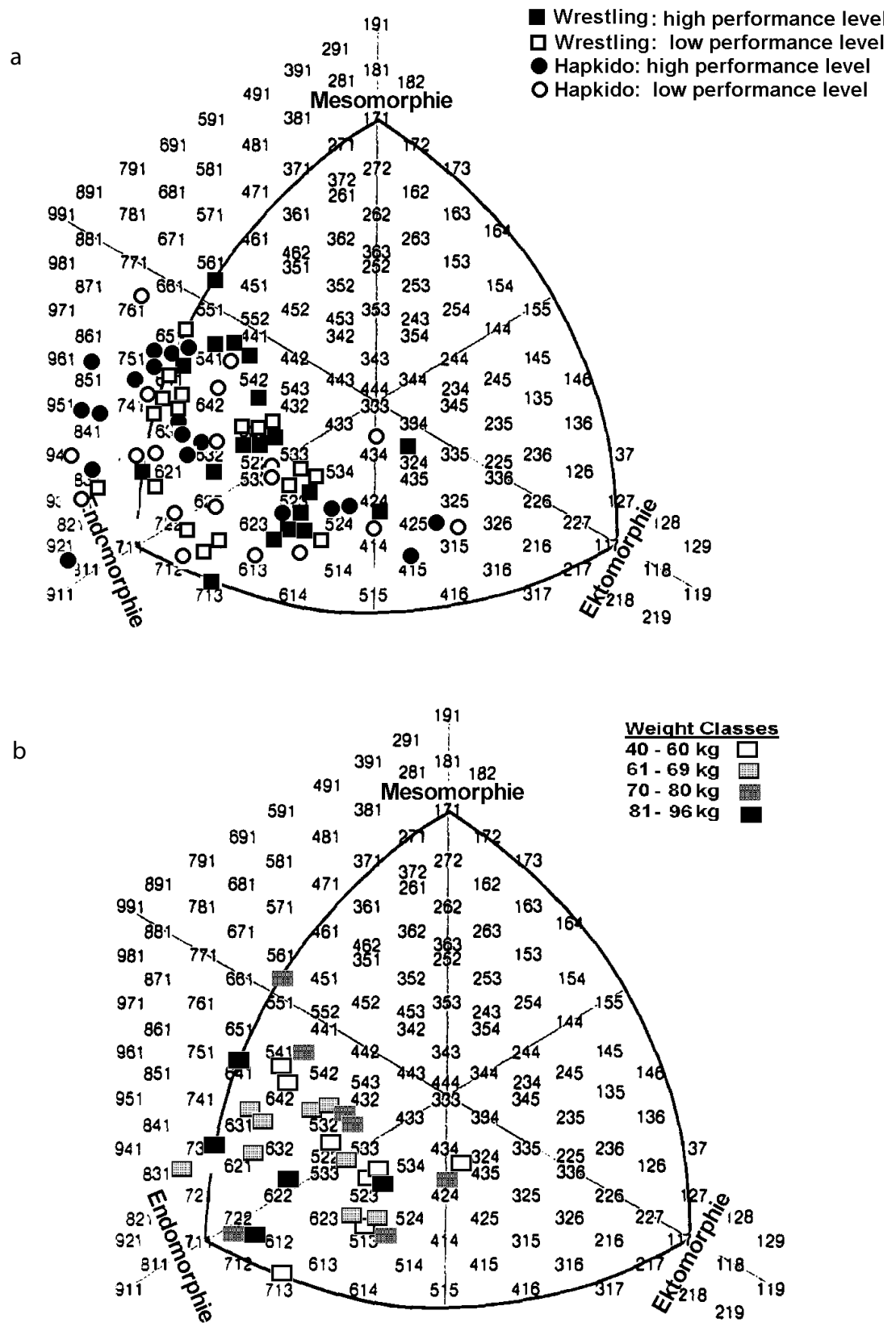

Fig. 3a+b. Male constitutional types of wrestlers and Hapkido fighters in the somatochart after Heath \& Carter (1967): a) first somatochart: wrestling vs. Hapkido; b) second somatochart: wrestling differentiated according to weight classes. 
The sports anthropometric parameters of male wrestlers and hapkidoin of different performance levels are listed in Table 1, the sports anthropometric parameters of male wrestlers, differentiated according to weight classes, are listed in Table 2.

Table 1. Sports anthropometric parameters of male wrestlers and hapkidoin of different performance levels

\begin{tabular}{|c|c|c|c|c|}
\hline Parameter & $\begin{array}{l}\text { Hapkido } \\
\text { Higher Class }\end{array}$ & $\begin{array}{l}\text { Hapkido } \\
\text { Lower Class }\end{array}$ & $\begin{array}{l}\text { Wrestling } \\
\text { Higher Class }\end{array}$ & $\begin{array}{l}\text { Wrestling } \\
\text { Lower Class }\end{array}$ \\
\hline Age (years) & $33.6 \pm 11.9$ & $30.8 \pm 11.7$ & $18.9 \pm 3.1$ & $20.9 \pm 6.2$ \\
\hline Height (Vertex; cm) & $176.8 \pm 6.8$ & $180.1 \pm 7.6$ & $170.4 \pm 8.6$ & $169.5 \pm 8.4$ \\
\hline Gnathion $(\mathrm{cm})$ & $153.2 \pm 6.5$ & $156.2 \pm 7.6$ & $147.2 \pm 8.0$ & $145.9 \pm 7.7$ \\
\hline Suprasternale $(\mathrm{cm})$ & $144.2 \pm 6.3$ & $147.0 \pm 6.4$ & $144.2 \pm 6.3$ & $147.0 \pm 6.4$ \\
\hline Acromiale $(\mathrm{cm})$ & $146.8 \pm 6.9$ & $149.7 \pm 6.8$ & $141.5 \pm 8.1$ & $140.5 \pm 7.4$ \\
\hline Radiale (cm) & $110.0 \pm 6.2$ & $113.9 \pm 5.7$ & $106.8 \pm 6.0$ & $105.4 \pm 6.4$ \\
\hline Stylion (cm) & $85.8 \pm 5.2$ & $88.3 \pm 4.9$ & $82.5 \pm 5.3$ & $81.8 \pm 5.3$ \\
\hline Dactylion $(\mathrm{cm})$ & $65.6 \pm 4.2$ & $69.6 \pm 5.3$ & $62.4 \pm 4.0$ & $62.9 \pm 6.3$ \\
\hline Iliocristale (cm) & $106.1 \pm 4.9$ & $109.9 \pm 5.6$ & $104.0 \pm 5.7$ & $103.2 \pm 5.7$ \\
\hline Iliospinale (cm) & $99.7 \pm 5.1$ & $102.7 \pm 5.6$ & $96.4 \pm 5.9$ & $95.8 \pm 5.4$ \\
\hline Tibiale $(\mathrm{cm})$ & $47.4 \pm 2.6$ & $49.0 \pm 2.4$ & $46.4 \pm 3.7$ & $45.4 \pm 3.8$ \\
\hline Sphyrion (cm) & $6.8 \pm 1.0$ & $6.2 \pm 0.9$ & $6.2 \pm 1.1$ & $6.3 \pm 1.4$ \\
\hline Sitting height $(\mathrm{cm})$ & $90.9 \pm 3.4$ & $92.9 \pm 3.0$ & $88.1 \pm 3.7$ & $87.6 \pm 4.8$ \\
\hline Arm span $(\mathrm{cm})$ & $182.2 \pm 7.6$ & $182.3 \pm 8.1$ & $175.8 \pm 9.3$ & $175.2 \pm 9.1$ \\
\hline Shoulder width $(\mathrm{cm})$ & $34.9 \pm 3.9$ & $33.6 \pm 2.5$ & $31.4 \pm 2.5$ & $31.2 \pm 2.4$ \\
\hline Chest width $(\mathrm{cm})$ & $30.9 \pm 3.6$ & $31.1 \pm 2.9$ & $29.4 \pm 2.3$ & $29.7 \pm 3.1$ \\
\hline Chest depth $(\mathrm{cm})$ & $21.5 \pm 2.3$ & $21.9 \pm 3.9$ & $20.3 \pm 1.5$ & $20.4 \pm 2.3$ \\
\hline Pelvis width $(\mathrm{cm})$ & $27.5 \pm 2.5$ & $27.7 \pm 2.9$ & $25.3 \pm 1.4$ & $25.8 \pm 2.7$ \\
\hline Spinal distance $(\mathrm{cm})$ & $23.8 \pm 2.5$ & $24.4 \pm 2.2$ & $23.2 \pm 2.8$ & $23.9 \pm 2.5$ \\
\hline Epiphysis width Femur (cm) & $7.6 \pm 1.4$ & $7.6 \pm 1.5$ & $6.3 \pm 0.7$ & $6.6 \pm 0.9$ \\
\hline Epiphysis width Humerus (cm) & $6.9 \pm 0.5$ & $7.0 \pm 0.9$ & $6.9 \pm 0.6$ & $6.8 \pm 0.7$ \\
\hline Hand breadth $(\mathrm{cm})$ & $8.2 \pm 0.8$ & $7.9 \pm 0.8$ & $8.3 \pm 1.6$ & $7.9 \pm 0.6$ \\
\hline Middle finger length (cm) & $9.7 \pm 0.7$ & $9.6 \pm 0.9$ & $8.8 \pm 0.8$ & $9.1 \pm 0.8$ \\
\hline $\begin{array}{l}\text { Anthropometric foot length } \\
\text { relieved }\end{array}$ & $25.8 \pm 1.3$ & $26.0 \pm 1.3$ & $25.4 \pm 1.5$ & $25.4 \pm 1.7$ \\
\hline $\begin{array}{l}\text { Anthropometric foot length } \\
\text { loaded }\end{array}$ & $26.1 \pm 1.3$ & $26.3 \pm 1.4$ & $25.7 \pm 1.4$ & $25.9 \pm 1.7$ \\
\hline
\end{tabular}




\begin{tabular}{|c|c|c|c|c|}
\hline Parameter & $\begin{array}{l}\text { Hapkido } \\
\text { Higher Class }\end{array}$ & $\begin{array}{l}\text { Hapkido } \\
\text { Lower Class }\end{array}$ & $\begin{array}{c}\text { Wrestling } \\
\text { Higher Class }\end{array}$ & $\begin{array}{l}\text { Wrestling } \\
\text { Lower Class }\end{array}$ \\
\hline Technological foot length & $20.9 \pm 1.6$ & $20.9 \pm 2.1$ & $20.8 \pm 1.4$ & $20.8 \pm 1.5$ \\
\hline Height of head & $23.6 \pm 1.5$ & $23.9 \pm 1.4$ & $23.2 \pm 1.6$ & $23.7 \pm 1.5$ \\
\hline Neck length & $9.0 \pm 1.7$ & $9.2 \pm 2.2$ & $9.0 \pm 2.1$ & $8.7 \pm 1.5$ \\
\hline Arm length & $81.1 \pm 3.6$ & $80.1 \pm 5.8$ & $79.4 \pm 5.2$ & $77.5 \pm 5.5$ \\
\hline Upper and lower arm length & $61.0 \pm 2.7$ & $61.4 \pm 3.2$ & $59.0 \pm 3.2$ & $59.2 \pm 2.7$ \\
\hline Upper arm length & $36.7 \pm 2.5$ & $35.7 \pm 2.1$ & $34.6 \pm 2.9$ & $35.1 \pm 2.1$ \\
\hline Lower arm length & $25.1 \pm 1.8$ & $25.6 \pm 1.6$ & $24.4 \pm 1.8$ & $23.8 \pm 1.8$ \\
\hline Hand length & $20.1 \pm 1.7$ & $19.7 \pm 1.6$ & $20.1 \pm 2.1$ & $19.8 \pm 2.1$ \\
\hline Morphologic leg length & $94.7 \pm 5.1$ & $97.7 \pm 5.6$ & $92.9 \pm 5.9$ & $92.0 \pm 4.7$ \\
\hline Physiognomic leg length & $85.8 \pm 5.8$ & $87.3 \pm 4.9$ & $82.3 \pm 5.6$ & $81.8 \pm 4.9$ \\
\hline Thigh and lower leg length & $88.9 \pm 4.6$ & $92.6 \pm 5.1$ & $87.2 \pm 6.2$ & $85.9 \pm 5.0$ \\
\hline Thigh length & $48.5 \pm 3.2$ & $49.8 \pm 3.7$ & $47.3 \pm 5.8$ & $46.7 \pm 3.5$ \\
\hline Lower leg length & $40.6 \pm 2.3$ & $42.8 \pm 2.0$ & $40.3 \pm 3.6$ & $39.1 \pm 3.6$ \\
\hline Heel width & $6.1 \pm 0.6$ & $6.1 \pm 0.5$ & $5.9 \pm 0.3$ & $5.9 \pm 0.3$ \\
\hline Foot width & $10.2 \pm 0.7$ & $10.3 \pm 0.6$ & $9.5 \pm 0.7$ & $9.7 \pm 0.8$ \\
\hline Neck circumference $(\mathrm{cm})$ & $38.1 \pm 2.5$ & $39.1 \pm 2.3$ & $40.2 \pm 1.8$ & $38.7 \pm 3.2$ \\
\hline $\begin{array}{l}\text { Chest circumference } \\
\text { (respiratory centre, cm) }\end{array}$ & $94.9 \pm 8.1$ & $96.9 \pm 9.9$ & $92.9 \pm 6.1$ & $96.9 \pm 26.7$ \\
\hline $\begin{array}{l}\text { Chest circumference in } \\
\text { inspiration }(\mathrm{cm})\end{array}$ & $100.6 \pm 7.2$ & $102.6 \pm 8.9$ & $97.6 \pm 5.9$ & $97.9 \pm 7.8$ \\
\hline $\begin{array}{l}\text { Chest circumference in } \\
\text { exspiration }(\mathrm{cm})\end{array}$ & $92.4 \pm 8.3$ & $94.3 \pm 9.2$ & $90.6 \pm 5.9$ & $89.8 \pm 7.6$ \\
\hline Waist circumference $(\mathrm{cm})$ & $83.9 \pm 9.5$ & $84.7 \pm 10.8$ & $77.3 \pm 4.5$ & $77.3 \pm 6.9$ \\
\hline Pelvis circumference $(\mathrm{cm})$ & $89.6 \pm 8.6$ & $90.4 \pm 9.5$ & $84.8 \pm 8.5$ & $86.2 \pm 8.6$ \\
\hline $\begin{array}{l}\text { Upper arm circumference in } \\
\text { flexion }(\mathrm{cm})\end{array}$ & $33.9 \pm 2.9$ & $33.2 \pm 2.9$ & $34.9 \pm 2.7$ & $33.3 \pm 4.2$ \\
\hline $\begin{array}{l}\text { Upper arm circumference } \\
\text { extension }(\mathrm{cm})\end{array}$ & $30.4 \pm 2.9$ & $29.9 \pm 2.7$ & $30.8 \pm 2.8$ & $29.7 \pm 3.6$ \\
\hline $\begin{array}{l}\text { Forearm circumf. maximum } \\
\text { (cm), dominant side }\end{array}$ & $27.3 \pm 1.9$ & $27.5 \pm 2.1$ & $28.1 \pm 1.6$ & $27.6 \pm 2.7$ \\
\hline $\begin{array}{l}\text { Forearm circumference } \\
\text { minimum }(\mathrm{cm})\end{array}$ & $17.3 \pm 1.3$ & $17.4 \pm 1.2$ & $17.7 \pm 1.2$ & $17.4 \pm 1.2$ \\
\hline Hand circumference $(\mathrm{cm})$ & $20.6 \pm 1.2$ & $20.4 \pm 1.5$ & $20.9 \pm 1.2$ & $20.3 \pm 1.4$ \\
\hline Thigh circumference $(\mathrm{cm})$ & $54.9 \pm 4.0$ & $54.8 \pm 4.4$ & $54.5 \pm 3.5$ & $53.1 \pm 4.9$ \\
\hline Calf circumference (cm) & $37.9 \pm 2.7$ & $37.6 \pm 3.1$ & $35.7 \pm 2.2$ & $35.9 \pm 3.3$ \\
\hline $\begin{array}{l}\text { Lower leg circumference } \\
\text { minimum }(\mathrm{cm})\end{array}$ & $25.3 \pm 2.4$ & $25.0 \pm 1.8$ & $23.3 \pm 1.8$ & $23.6 \pm 1.8$ \\
\hline
\end{tabular}




\begin{tabular}{|c|c|c|c|c|}
\hline Parameter & $\begin{array}{l}\text { Hapkido } \\
\text { Higher Class }\end{array}$ & $\begin{array}{l}\text { Hapkido } \\
\text { Lower Class }\end{array}$ & $\begin{array}{l}\text { Wrestling } \\
\text { Higher Class }\end{array}$ & $\begin{array}{l}\text { Wrestling } \\
\text { Lower Class }\end{array}$ \\
\hline Foot circumference $(\mathrm{cm})$ & $25.9 \pm 1.6$ & $26.1 \pm 1.4$ & $24.8 \pm 1.1$ & $24.8 \pm 1.9$ \\
\hline Morphological facial height $(\mathrm{cm})$ & $12.6 \pm 1.0$ & $12.7 \pm 0.9$ & $12.5 \pm 1.1$ & $12.3 \pm 0.9$ \\
\hline Zygomatic breadth (cm) & $12.5 \pm 1.2$ & $12.8 \pm 1.1$ & $12.1 \pm 0.8$ & $12.1 \pm 1.1$ \\
\hline Subscapular skinfold (mm) & $20.8 \pm 7.6$ & $19.9 \pm 8.0$ & $15.7 \pm 4.9$ & $15.3 \pm 4.4$ \\
\hline Triceps skinfold (mm) & $23.1 \pm 5.9$ & $22.3 \pm 5.6$ & $20.5 \pm 3.9$ & $24.7 \pm 4.2$ \\
\hline Forearm skinfold (mm) & $9.8 \pm 2.7$ & $9.6 \pm 2.7$ & $14.1 \pm 2.1$ & $14.4 \pm 2.3$ \\
\hline Suprailiac skinfold (mm) & $26.5 \pm 11.3$ & $25.7 \pm 9.2$ & $16.9 \pm 5.2$ & $18.8 \pm 5.9$ \\
\hline Thigh skinfold (mm) & $20.3 \pm 7.4$ & $17.6 \pm 5.5$ & $19.9 \pm 4.8$ & $21.8 \pm 8.9$ \\
\hline Calf skinfold (mm) & $17.4 \pm 6.9$ & $17.3 \pm 6.2$ & $20.2 \pm 4.7$ & $21.5 \pm 5.2$ \\
\hline $\begin{array}{l}\text { Body fat percentage } \\
\text { (calipermetry;\%) }\end{array}$ & $27.7 \pm 9.5$ & $24.5 \pm 8.6$ & $18.6 \pm 4.5$ & $19.9 \pm 5.2$ \\
\hline Body fat percentage (BIA;\%) & $19.1 \pm 5.2$ & $18.2 \pm 7.0$ & $18.7 \pm 8.2$ & $19.6 \pm 9.3$ \\
\hline Plastik-Index after Conrad & $83.3 \pm 5.5$ & $81.4 \pm 5.2$ & $80.5 \pm 4.5$ & $79.2 \pm 5.5$ \\
\hline Metrik-Index after Conrad & $-0.1 \pm 0.9$ & $-0.3 \pm 0.9$ & $-0.5 \pm 0.4$ & $-0.4 \pm 0.6$ \\
\hline Pyknomorphy after Knußmann & $-6.8 \pm 3.3$ & $-5.9 \pm 1.5$ & $-4.8 \pm 1.5$ & $-4.8 \pm 1.8$ \\
\hline Makrosomia after Knußmann & $-2.0 \pm 2.5$ & $-1.7 \pm 3.1$ & $-3.3 \pm 3.1$ & $-3.3 \pm 2.6$ \\
\hline Endomorphy after Parnell & $5.3 \pm 0.8$ & $5.1 \pm 0.5$ & $5.1 \pm 0.7$ & $5.3 \pm 0.4$ \\
\hline Mesomorphy after Parnell & $2.5 \pm 1.2$ & $2.3 \pm 1.1$ & $2.9 \pm 1.4$ & $2.8 \pm 1.0$ \\
\hline Ectomorphy after Parnell & $3.9 \pm 1.4$ & $4.0 \pm 1.0$ & $2.9 \pm 0.9$ & $3.1 \pm 0.9$ \\
\hline $\begin{array}{l}\text { Endomorphy after } \\
\text { Heath\&Carter }\end{array}$ & $6.2 \pm 1.7$ & $6.0 \pm 1.6$ & $5.2 \pm 0.9$ & $5.7 \pm 0.7$ \\
\hline $\begin{array}{l}\text { Mesomorphy after } \\
\text { Heath\&Carter }\end{array}$ & $3.0 \pm 1.5$ & $2.5 \pm 1.3$ & $2.6 \pm 1.4$ & $2.6 \pm 1.0$ \\
\hline Ectomorphy after Heath\&Carter & $2.0 \pm 1.4$ & $2.1 \pm 1.1$ & $1.6 \pm 0.8$ & $1.8 \pm 0.9$ \\
\hline Body weight (kg) & $77.9 \pm 12.5$ & $81.5 \pm 14.6$ & $71.3 \pm 10.5$ & $70.3 \pm 13.9$ \\
\hline $\operatorname{BMI}\left(\mathrm{kg} / \mathrm{m}^{2}\right)$ & $24.6 \pm 3.5$ & $25.0 \pm 3.1$ & $24.4 \pm 2.1$ & $24.1 \pm 2.9$ \\
\hline Pelidisi-Index (kg/cm) & $100.9 \pm 6.2$ & $100.2 \pm 4.2$ & $101.2 \pm 2.7$ & $101.0 \pm 3.3$ \\
\hline Quetelet-Index (g/cm) & $4.4 \pm 0.6$ & $4.5 \pm 0.7$ & $4.2 \pm 0.5$ & $4.1 \pm 0.6$ \\
\hline Lean Body Mass LBM (kg) & $77.8 \pm 12.5$ & $81.5 \pm 14.5$ & $71.3 \pm 10.5$ & $70.3 \pm 13.9$ \\
\hline AKS-Index (BIA) & $1.1 \pm 0.3$ & $0.9 \pm 0.2$ & $1.2 \pm 0.2$ & $1.2 \pm 0.2$ \\
\hline AKS-Index (Caliper) & $1.0 \pm 0.2$ & $1.0 \pm 0.2$ & $1.2 \pm 0.2$ & $1.2 \pm 0.1$ \\
\hline Body Surface $\left(\mathrm{m}^{2}\right)$ & $1.9 \pm 0.2$ & $2.0 \pm 0.2$ & $1.8 \pm 0.2$ & $1.8 \pm 0.2$ \\
\hline Rohrer-Index $\left(\mathrm{g} / \mathrm{cm}^{3}\right)$ & $1.4 \pm 0.2$ & $1.4 \pm 0.2$ & $1.4 \pm 0.1$ & $1.4 \pm 0.1$ \\
\hline Broca-Index (\%) & $0.7 \pm 14.1$ & $1.3 \pm 12.2$ & $1.4 \pm 8.4$ & $1.0 \pm 11.2$ \\
\hline $\begin{array}{l}\text { Height-Weight-Ratio } \\
\text { (inches/3. } \sqrt{\mathrm{lb} \text { ) }}\end{array}$ & $13.0 \pm 0.7$ & $13.0 \pm 0.5$ & $12.9 \pm 0.4$ & $12.9 \pm 0.4$ \\
\hline
\end{tabular}


Table 2. Sports anthropometric parameters of male wrestlers, differentiated according to weight classes

\begin{tabular}{|c|c|c|c|c|}
\hline $\begin{array}{l}\text { Parameter - male wrestlers - } \\
\text { weight classes }\end{array}$ & $40-60 \mathrm{~kg}$ & $61-69$ kg & $70-80 \mathrm{~kg}$ & $81-96 \mathrm{~kg}$ \\
\hline Age (years) & $17.6 \pm 3.6$ & $19.8 \pm 3.8$ & $19.8 \pm 4.1$ & $23.1 \pm 7.3$ \\
\hline Height (Vertex; cm) & $160.6 \pm 4.4$ & $168.1 \pm 3.7$ & $172.4 \pm 4.7$ & $181.6 \pm 3.2$ \\
\hline Gnathion $(\mathrm{cm})$ & $138.4 \pm 4.2$ & $144.6 \pm 4.1$ & $148.7 \pm 4.3$ & $157.3 \pm 3.8$ \\
\hline Suprasternale $(\mathrm{cm})$ & $129.8 \pm 3.6$ & $135.9 \pm 3.4$ & $140.2 \pm 3.6$ & $147.4 \pm 2.8$ \\
\hline Acromiale $(\mathrm{cm})$ & $132.8 \pm 4.0$ & $139.2 \pm 4.1$ & $143.3 \pm 4.4$ & $151.3 \pm 4.0$ \\
\hline Radiale $(\mathrm{cm})$ & $99.9 \pm 3.9$ & $104.9 \pm 2.6$ & $107.2 \pm 4.2$ & $114.6 \pm 3.0$ \\
\hline Stylion (cm) & $76.4 \pm 2.5$ & $81.1 \pm 2.5$ & $83.8 \pm 3.3$ & $89.1 \pm 2.8$ \\
\hline Dactylion $(\mathrm{cm})$ & $57.8 \pm 2.9$ & $61.8 \pm 1.4$ & $63.1 \pm 2.8$ & $69.8 \pm 5.3$ \\
\hline Iliocristale $(\mathrm{cm})$ & $98.3 \pm 3.3$ & $101.9 \pm 2.2$ & $104.9 \pm 4.4$ & $111.2 \pm 3.3$ \\
\hline Iliospinale $(\mathrm{cm})$ & $90.6 \pm 3.1$ & $94.4 \pm 2.6$ & $97.8 \pm 4.5$ & $103.4 \pm 2.7$ \\
\hline Tibiale $(\mathrm{cm})$ & $42.9 \pm 3.2$ & $45.7 \pm 2.2$ & $47.1 \pm 3.2$ & $48.8 \pm 4.0$ \\
\hline Sphyrion (cm) & $6.1 \pm 1.2$ & $5.7 \pm 0.6$ & $6.3 \pm 0.9$ & $7.1 \pm 1.9$ \\
\hline Sitting height $(\mathrm{cm})$ & $83.5 \pm 3.6$ & $87.3 \pm 2.2$ & $88.9 \pm 2.5$ & $93.1 \pm 1.9$ \\
\hline Arm span $(\mathrm{cm})$ & $166.6 \pm 5.1$ & $172.5 \pm 6.3$ & $178.2 \pm 4.4$ & $187.9 \pm 4.6$ \\
\hline Shoulder width $(\mathrm{cm})$ & $29.5 \pm 1.8$ & $30.9 \pm 1.7$ & $31.5 \pm 2.1$ & $34.1 \pm 1.8$ \\
\hline Chest width $(\mathrm{cm})$ & $26.7 \pm 1.5$ & $29.1 \pm 1.2$ & $30.5 \pm 2.1$ & $32.9 \pm 1.3$ \\
\hline Chest depth $(\mathrm{cm})$ & $18.8 \pm 1.6$ & $20.1 \pm 1.8$ & $20.6 \pm 0.8$ & $22.3 \pm 1.9$ \\
\hline Pelvis width (cm) & $24.4 \pm 1.4$ & $25.2 \pm 0.9$ & $25.4 \pm 2.4$ & $27.9 \pm 2.0$ \\
\hline Spinal distance $(\mathrm{cm})$ & $22.9 \pm 2.7$ & $22.6 \pm 3.1$ & $23.6 \pm 1.6$ & $25.8 \pm 2.2$ \\
\hline Epiphysis width Humerus (cm) & $6.6 \pm 0.7$ & $6.9 \pm 0.5$ & $6.8 \pm 0.6$ & $7.4 \pm 0.4$ \\
\hline Epiphysis width Femur (cm) & $8.1 \pm 0.6$ & $8.3 \pm 0.6$ & $8.3 \pm 0.9$ & $9.1 \pm 0.9$ \\
\hline Middle finger length $(\mathrm{cm})$ & $6.4 \pm 0.5$ & $7.9 \pm 0.8$ & $7.9 \pm 0.3$ & $9.0 \pm 0.9$ \\
\hline Anthropometric foot length relieved & $23.9 \pm 0.9$ & $25.0 \pm 1.0$ & $25.9 \pm 1.1$ & $27.3 \pm 1.0$ \\
\hline Anthropometric foot length loaded & $24.5 \pm 0.7$ & $25.2 \pm 1.0$ & $26.2 \pm 1.1$ & $27.8 \pm 1.2$ \\
\hline Technological foot length & $19.3 \pm 0.9$ & $20.5 \pm 1.2$ & $20.9 \pm 1.0$ & $22.6 \pm 1.2$ \\
\hline Height of head & $22.2 \pm 1.6$ & $23.6 \pm 1.2$ & $23.7 \pm 1.5$ & $24.3 \pm 1.6$ \\
\hline Neck length & $8.6 \pm 1.4$ & $8.7 \pm 2.6$ & $8.5 \pm 1.5$ & $9.9 \pm 1.5$ \\
\hline Arm length & $75.0 \pm 2.2$ & $77.5 \pm 3.5$ & $80.1 \pm 2.2$ & $82.3 \pm 9.4$ \\
\hline Upper and lower arm length & $57.0 \pm 2.7$ & $58.2 \pm 2.4$ & $59.9 \pm 1.8$ & $62.1 \pm 2.1$ \\
\hline Upper arm length & $32.8 \pm 2.6$ & $34.3 \pm 1.8$ & $36.1 \pm 1.7$ & $36.6 \pm 2.2$ \\
\hline Lower arm length & $23.6 \pm 2.2$ & $23.8 \pm 1.4$ & $23.9 \pm 1.8$ & $25.6 \pm 1.2$ \\
\hline Hand length & $18.6 \pm 1.5$ & $19.3 \pm 1.8$ & $20.2 \pm 1.5$ & $22.3 \pm 1.9$ \\
\hline
\end{tabular}




\begin{tabular}{|c|c|c|c|c|}
\hline $\begin{array}{l}\text { Parameter - male wrestlers - } \\
\text { weight classes }\end{array}$ & $40-60 \mathrm{~kg}$ & $61-69 \mathrm{~kg}$ & $70-80 \mathrm{~kg}$ & $81-96 \mathrm{~kg}$ \\
\hline Morphologic leg length & $87.4 \pm 2.8$ & $91.8 \pm 4.9$ & $93.7 \pm 4.0$ & $98.6 \pm 2.4$ \\
\hline Physiognomic leg length & $77.1 \pm 3.8$ & $80.8 \pm 2.6$ & $83.6 \pm 3.4$ & $88.4 \pm 3.7$ \\
\hline Thigh and lower leg length & $81.3 \pm 3.8$ & $86.4 \pm 4.9$ & $87.8 \pm 3.9$ & $92.5 \pm 4.1$ \\
\hline Thigh length & $44.4 \pm 2.8$ & $46.5 \pm 6.8$ & $47.2 \pm 2.1$ & $50.9 \pm 4.1$ \\
\hline Lower leg length & $36.9 \pm 3.4$ & $39.9 \pm 2.4$ & $40.7 \pm 2.9$ & $41.7 \pm 4.0$ \\
\hline Heel width & $5.8 \pm 0.4$ & $5.9 \pm 0.3$ & $5.8 \pm 0.3$ & $6.1 \pm 0.2$ \\
\hline Foot width & $9.3 \pm 0.8$ & $9.6 \pm 0.7$ & $9.6 \pm 0.7$ & $10.0 \pm 0.9$ \\
\hline Neck circumference $(\mathrm{cm})$ & $36.4 \pm 2.1$ & $40.1 \pm 1.8$ & $39.9 \pm 1.1$ & $42.4 \pm 1.0$ \\
\hline $\begin{array}{l}\text { Chest circumference (respiratory } \\
\text { centre, } \mathrm{cm} \text { ) }\end{array}$ & $83.8 \pm 3.5$ & $91.4 \pm 2.2$ & $95.0 \pm 2.9$ & $102.0 \pm 3.2$ \\
\hline Chest circumference in inspiration $(\mathrm{cm})$ & $89.6 \pm 3.3$ & $96.4 \pm 2.2$ & $100.3 \pm 2.7$ & $107.1 \pm 3.3$ \\
\hline Chest circumference in exspiration $(\mathrm{cm})$ & $82.2 \pm 3.7$ & $89.5 \pm 1.9$ & $92.4 \pm 2.8$ & $99.3 \pm 3.2$ \\
\hline Waist circumference $(\mathrm{cm})$ & $71.8 \pm 3.3$ & $76.1 \pm 2.3$ & $77.6 \pm 4.1$ & $85.8 \pm 2.4$ \\
\hline Pelvis circumference $(\mathrm{cm})$ & $81.4 \pm 5.2$ & $84.1 \pm 7.6$ & $86.4 \pm 6.7$ & $91.8 \pm 12.0$ \\
\hline Upper arm circumference in flexion $(\mathrm{cm})$ & $30.4 \pm 2.8$ & $34.5 \pm 2.6$ & $34.2 \pm 2.1$ & $38.4 \pm 1.9$ \\
\hline $\begin{array}{l}\text { Upper arm circumference } \\
\text { extension }(\mathrm{cm})\end{array}$ & $26.7 \pm 2.1$ & $30.2 \pm 2.1$ & $30.9 \pm 1.7$ & $34.3 \pm 1.8$ \\
\hline $\begin{array}{l}\text { Forearm circumf. maximum }(\mathrm{cm}) \text {, } \\
\text { dominant side }\end{array}$ & $25.4 \pm 1.3$ & $27.8 \pm 0.8$ & $28.3 \pm 1.4$ & $30.8 \pm 1.2$ \\
\hline Forearm circumference minimum $(\mathrm{cm})$ & $16.5 \pm 0.6$ & $17.7 \pm 1.0$ & $17.7 \pm 1.1$ & $18.7 \pm 0.9$ \\
\hline Hand circumference $(\mathrm{cm})$ & $19.9 \pm 1.2$ & $20.7 \pm 0.8$ & $20.3 \pm 1.2$ & $22.0 \pm 1.3$ \\
\hline Thigh circumference $(\mathrm{cm})$ & $49.4 \pm 3.7$ & $54.0 \pm 2.2$ & $54.6 \pm 1.9$ & $58.6 \pm 3.6$ \\
\hline Calf circumference $(\mathrm{cm})$ & $33.2 \pm 2.0$ & $35.6 \pm 2.1$ & $36.5 \pm 2.0$ & $38.9 \pm 2.1$ \\
\hline Lower leg circumference minimum (cm) & $21.9 \pm 1.6$ & $23.2 \pm 1.1$ & $23.7 \pm 1.1$ & $25.5 \pm 1.4$ \\
\hline Foot circumference $(\mathrm{cm})$ & $23.7 \pm 1.5$ & $24.8 \pm 0.9$ & $24.7 \pm 1.2$ & $26.3 \pm 1.8$ \\
\hline Morphological facial height (cm) & $11.9 \pm 0.7$ & $12.3 \pm 1.2$ & $12.6 \pm 1.0$ & $12.9 \pm 0.8$ \\
\hline Zygomatic breadth (cm) & $11.6 \pm 1.2$ & $12.2 \pm 0.7$ & $12.2 \pm 0.7$ & $12.6 \pm 1.1$ \\
\hline Subscapular skinfold (mm) & $13.0 \pm 4.3$ & $14.4 \pm 3.0$ & $16.2 \pm 3.9$ & $19.3 \pm 5.2$ \\
\hline Triceps skinfold (mm) & $21.2 \pm 4.9$ & $21.2 \pm 5.4$ & $23.7 \pm 3.1$ & $24.8 \pm 3.9$ \\
\hline Forearm skinfold (mm) & $14.8 \pm 2.6$ & $14.3 \pm 2.7$ & $13.6 \pm 1.7$ & $14.3 \pm 1.4$ \\
\hline Suprailiac skinfold (mm) & $17.0 \pm 5.4$ & $15.3 \pm 2.8$ & $19.5 \pm 7.0$ & $20.1 \pm 5.5$ \\
\hline Thigh skinfold (mm) & $21.5 \pm 7.8$ & $21.0 \pm 7.1$ & $17.4 \pm 5.5$ & $24.7 \pm 7.1$ \\
\hline Calf skinfold (mm) & $21.2 \pm 5.4$ & $19.7 \pm 5.6$ & $19.5 \pm 3.5$ & $23.6 \pm 5.0$ \\
\hline Body fat percentage (calipermetry; \%) & $17.4 \pm 5.0$ & $17.7 \pm 2.3$ & $20.7 \pm 5.6$ & $22.0 \pm 4.8$ \\
\hline Body fat percentage (BIA;\%) & $20.2 \pm 11.6$ & $18.4 \pm 7.9$ & $19.6 \pm 9.8$ & $18.0 \pm 2.6$ \\
\hline
\end{tabular}




\begin{tabular}{|c|c|c|c|c|}
\hline $\begin{array}{l}\text { Parameter - male wrestlers - } \\
\text { weight classes }\end{array}$ & $40-60 \mathrm{~kg}$ & $61-69$ kg & $70-80$ kg & $81-96 \mathrm{~kg}$ \\
\hline Plastik-Index after Conrad & $74.7 \pm 2.8$ & $79.3 \pm 1.9$ & $80.2 \pm 3.3$ & $86.9 \pm 2.7$ \\
\hline Metrik-Index after Conrad & $-0.8 \pm 0.3$ & $-0.4 \pm 0.4$ & $-0.4 \pm 0.6$ & $-0.2 \pm 0.4$ \\
\hline Pyknomorphy after Knußmann & $-5.1 \pm 1.6$ & $-4.7 \pm 1.4$ & $-4.7 \pm 1.8$ & $-4.9 \pm 1.8$ \\
\hline Makrosomia after Knußmann & $-5.3 \pm 2.0$ & $-4.4 \pm 2.6$ & $-2.9 \pm 1.8$ & $0.3 \pm 0.9$ \\
\hline Endomorphy after Parnell & $5.1 \pm 0.8$ & $5.1 \pm 0.3$ & $5.4 \pm 0.6$ & $5.4 \pm 0.6$ \\
\hline Mesomorphy after Parnell & $2.6 \pm 1.4$ & $3.3 \pm 1.4$ & $2.6 \pm 1.0$ & $3.1 \pm 1.1$ \\
\hline Ectomorphy after Parnell & $3.4 \pm 1.0$ & $2.9 \pm 0.9$ & $2.8 \pm 0.9$ & $2.8 \pm 0.5$ \\
\hline Endomorphy after Heath\&Carter & $5.3 \pm 1.0$ & $5.1 \pm 0.7$ & $5.6 \pm 0.8$ & $5.8 \pm 0.8$ \\
\hline Mesomorphy after Heath\&Carter & $2.2 \pm 1.2$ & $2.8 \pm 1.6$ & $2.5 \pm 0.9$ & $3.2 \pm 1.0$ \\
\hline Ectomorphy after Heath\&Carter & $2.2 \pm 1.0$ & $1.6 \pm 0.7$ & $1.6 \pm 0.8$ & $1.3 \pm 0.5$ \\
\hline Body weight (kg) & $56.4 \pm 5.3$ & $68.2 \pm 1.7$ & $74.0 \pm 3.6$ & $89.3 \pm 4.3$ \\
\hline BMI $\left(\mathrm{kg} / \mathrm{m}^{2}\right)$ & $21.8 \pm 2.2$ & $24.0 \pm 1.7$ & $24.9 \pm 1.6$ & $26.9 \pm 1.4$ \\
\hline Pelidisi-Index $(\mathrm{kg} / \mathrm{cm})$ & $98.8 \pm 3.2$ & $100.9 \pm 2.8$ & $101.8 \pm 2.4$ & $101.8 \pm 2.4$ \\
\hline Quetelet-Index (g/cm) & $3.5 \pm 0.3$ & $4.1 \pm 0.2$ & $4.3 \pm 0.2$ & $4.9 \pm 0.2$ \\
\hline Lean Body Mass LBM (kg) & $56.4 \pm 5.3$ & $68.2 \pm 1.7$ & $74.0 \pm 3.6$ & $89.3 \pm 4.3$ \\
\hline AKS-Index (BIA) & $1.1 \pm 0.2$ & $1.2 \pm 0.2$ & $1.2 \pm 0.2$ & $1.2 \pm 0.2$ \\
\hline AKS-Index (Caliper) & $1.2 \pm 0.2$ & $1.2 \pm 0.2$ & $1.2 \pm 0.1$ & $1.1 \pm 0.1$ \\
\hline Body Surface $\left(\mathrm{m}^{2}\right)$ & $1.6 \pm 0.1$ & $1.8 \pm 0.0$ & $1.9 \pm 0.1$ & $2.1 \pm 0.1$ \\
\hline Rohrer-Index $\left(\mathrm{g} / \mathrm{cm}^{3}\right)$ & $1.4 \pm 0.2$ & $1.4 \pm 0.1$ & $1.5 \pm 0.1$ & $1.5 \pm 0.1$ \\
\hline Broca-Index (\%) & $-6.6 \pm 1.0$ & $0.5 \pm 6.9$ & $2.5 \pm 7.3$ & $9.9 \pm 4.4$ \\
\hline
\end{tabular}

\section{DISCUSSION}

On average, the mean age of wrestlers was lower (18.9 years in the higher performance class and 20.9 years in the lower performance class), compared to hapkidoin (33.5 years in the higher performance class, 30.8 years in the lower performance class). The differences are explained by the fact that wrestling is primarily exercised as a sport, hapkido rather as a hobby.

The wrestlers of both performance classes are considerably smaller (mean height $170 \mathrm{~cm}$ ) than the hapkidoin (high performance class $176.5 \mathrm{~cm}$, lower performance class $180 \mathrm{~cm}$ ). When comparing the weight classes of the wrestlers, the average body height increases continuously with increasing weight class in conjunction with most other anthropometric parameters. 
In the chessboard pattern graphic to Conrad, the hapkidoin appear rather moderately pyknomorphic as well as slender, the wrestlers are moderately leptomorphic and metrosome as well as smaller.

In Knussmann's typology the hapkidoin have a more muscular appearance. The wrestlers tend to microsomy and leptomorphy.

In Parnell's somatochart, the wrestlers have an average somatotype of 5 $3-3$, the hapkidoin of $5-3-4$.

In the somatochart of Heath \& Carter, an average somatotype of $6-3-2$ is shown for both combat sport collectives, whereby the wrestlers tend to align themselves to the endomorphic axis.

While hapkidoin have not yet been investigated, the average somatotypes of Judoka were 3.6 - 7 - 1.9 (Farmosi 1980), Karateka 2.6 - 5.2 - 2.6 (Claessens et al., 1986) and a mixture type (whu-shu, kung-fu, karate, judo) at 2.7 - $4.7-2.7$ (Gualdi-Russo et al., 1993).

In further studies the different wrestling styles like Freestyle and GraecoRoman style are to be investigated and compared.

\section{REFERENCES}

1. Claessens A., Beunen G., Lefevre J., Martens G., Wellens R. (1986). Body structure, somatotype, and motor fitness of top-class Belgian judoists and karateka: a comparative study. In Kinanthropometry III, ed. T. Reilly, J. Watkins, and J. Borms, 53-57. London: E. \& F.N. Spon.

2. Conrad K. (1963). Der Konstitutionstypus. Berlin: Springer.

3. Farmosi I. (1980). Body-composition, somatotype and some motor performance of judoists. Sports Med Phys Fitness, 20, 431-434.

4. Gualdi-Russo E., Graziani I. (1993). Anthropometric somatotype of Italian sport participants. J Sports Med Phys Fitness, 33, 282-291.

5. Heath B.H., Carter L.J.E. (1967). A modified somatotype method. Am J Phys Anthrop, 27, 57-74.

6. Heath B.H., Carter L.J.E. (1990). Somatotyping - development and applications. Cambridge Studies in Biological Anthropology. Redwood Press.

7. Knußmann R. (1996). Vergleichende Biologie des Menschen. Lehrbuch der Anthropologie. Stuttgart: Gustav Fischer.

8. Kretschmer E. (1921). Körperbau und Charakter. Berlin: Springer.

9. Martin R., Knußmann R. (1988). Anthropologie. Handbuch. Band I. Stuttgart: Fischer.

10. Parnell R.W. (1954). Somatotyping by physical anthropometry. Am J Phys Anthrop, 12, 209-239. 
11. Raschka C. (2006). Sportanthropologie. Köln: Sportverlag Strauß.

12. Tittel K., Wutscherk H. (1972). Sportanthropometrie. Leipzig: Barth.

Adress for correspondence

Prof. Dr.med. Dr.rer.nat. Dr.Sportwiss. Christoph Raschka

Institute of Sports Sciences, Julius-Maximilians-University, Germany

Judenbühlweg 11

D-97082 Würzburg

E-mail: christoph.raschka@uni-wuerzburg.de 